\title{
The $\rho$ meson mass spectrum using soft-wall QCD
}

\author{
Vlasios Petousis* \\ Institute Rudjer Boskovic. Bijencka-cesta 54, HR-10000 Zagreb, Croatia \\ E-mail: vlasios.petousisecern.ch, vpetouseirb.hr
}

\begin{abstract}
Meson spectroscopy within the frame of the soft-wall AdS/QCD, became one of the most interesting topics of particle physics in the last six years. In this work we analyze the mass spectrum of the $\rho$ vector meson, using a very simple modification of the background dilaton gauge field within the soft-wall AdS/QCD model. Aim of this work is that using a positive z-depended dilaton gauge field, even with that simple modification, the correct Regge trajectories can be reproduced without problems with massless modes in the vector sector. To reinforce the results, we compare the proposed model with the experimental data. This comparison returns an error less than $1 \%$ for the high $\rho$ masses.
\end{abstract}

Sixth International Conference on Quarks and Nuclear Physics,

April 16-20, 2012

Ecole Polytechnique, Palaiseau, Paris

\footnotetext{
* Speaker.

${ }^{\dagger}$ Poster Presenter.
} 


\section{Introduction}

Since the 1970's the progress towards to understand the strong interaction has developed surprisingly. Quantum Chromodynamics (QCD) has established as a very successful quantum field theory description of the strong interaction and is by now well tested experimentally. The matter degrees of freedom in QCD consist of quarks transforming in representation of a non-abelian SU(3) gauge theory. Interactions are mediated by gauge bosons, the gluon fields, in the adjoint representation of SU(3). The theory has been shown to be asymptotically free. According to this at large energy scales or equivalent at very short distances the quarks becomes weakly interacting. Contrary at long distances or low energies the force becomes stronger. As result the quarks are confined into bound states, the hadrons. Also in addition the large mass of the quarks, breaks the chiral symmetry, mixing left and right handed quarks. One decade earlier the discovery of QCD in 1960, a radical theory has originated, was the string theory. Found that the hadron spectra contains Regge trajectories, a step further was the realization that these trajectories can be reproduced by a rotating relativistic string. Those days the four dimensional string theories lead to non physical modes like tachyons and massless vector particles. So the string theory rejected as a theory which can describe the strong interaction and took another way. String theory today is a potentially promising candidate for the unified theory of all fundamental interactions. The success both of QCD and string theory is enormous but many unsolved problems remains an open issues until today. For the QCD despite the help of the powerful computing (lattice QCD) the low energy mechanism for the confinement and chiral symmetry breaking, remains blur. The string theory in that approach as mentioned before could be a favorable potential candidate, but is more than desirable the connection with the experiments. Many open issues and questions it seems that have found a hint of understanding since 1990's. One of the relations between superstring theory and QCD done on 1995, introducing the concept of D-branes. In 1997 a radical idea about a dual interpretation of D-branes putted forward by Juan Maldacena [1]. In a few words one can say that in Maldacena's duality we can see the matching between a symmetric strongly coupled large $\mathrm{N}$ gauge theory and weakly coupled supergravity theory. The gauge theory is a $3+1$ dimensional theory which comes out from a basic D3 brane configuration. $\mathrm{N}$ number of branes generates an $\mathrm{SU}(\mathrm{N})$ gauge theory in the lowest energy limit and to have a supergravity weakly coupled the theory needs a large number of branes. The introduction of QCD like new theories is necessary to break supersymmetry and remove conformal invariance as well to introduce quark fields. String theory models have inspired phenomenological approaches to QCD gives us the AdS/QCD. More concretely the AdS/CFT correspondence is a duality between the type IIB tring theory defined on the $A d S_{5} \times S^{5}$ space and a $N=4$ super Yang-Mills theory with gauge group $S U\left(N_{c}\right)$ for large $N_{c}$. Little later proposed that the correspondence could be generalized as an a equivalence between a theory defined an $A d S_{d+1} \times L$ and a conformal field theory living on the flat boundary $M_{D}$ of the AdS space [2]. This has provided a new hope in the understanding of strong interaction processes by string inspired approaches. Two main ways followed to achieve this goal. The first so-called top-down approach, for this one starts from string theory and tries to derive a low-energy QCD like theory on $M_{D}$ through a compactifications of the extra dimensions. The second is the bottom-up approach in which one starts from 4D QCD and try to construct higher dimensional dual theory [3]. Both approaches must break conformal invariance, since QCD is not a conformal theory [4] and 
to achieved confinement. The bottom-up approach has two ways to achieved also. The first is the hard-wall model which uses a five dimensional "AdS-slice" with the fifth holographic coordinate $\mathrm{z}$ varying up a $z_{\max }$. Another proposal to break conformal invariance can be done introducing at 5D AdS space, a background dilaton field. The latter model named soft-wall model [5],[6]. The backbone of this paper organized as follows: in section 2 presented a sort basic review of the softwall model and in section 3 introduced the proposed dilaton gauge field. In section 4 the masses spectrum of $\rho$ vector meson calculated and comes in comparison with the experiment. Finally this paper ends with the conclusions and the appropriate bibliography.

\section{A review in Soft-Wall AdS/QCD}

To describe the model one can start from QCD and constructs its gravity dual theory. A good point to start is the Dirac Lagrangian which governs the motion of quarks. This Lagrangian has a global $S U\left(N_{f}\right)_{L} \times S U\left(N_{f}\right)_{R}$ symmetry in a massless limit. The chiral symmetry is broken by the mass term. Additionally there is another source for this that so-called quarks condensate and that breaks spontaneously the chiral symmetry. Also the spectra of mesons in low-energy QCD are linear and the quarks are confined inside the hadrons. This linear confinement has to be realized in any realistic model of low-energy QCD. Building a holographic dual of QCD one must start with these basic ingredients. Each global symmetry of QCD becomes a local symmetry in the gravity (holographic) side. Thus, in a gravity side, there is one left-handed gauge vector field $A_{L}$ for the global $S U\left(N_{f}\right)_{L}$ symmetry of QCD and one right-handed gauge vector field $A_{R}$ for the global $S U\left(N_{f}\right)_{R}$ symmetry of QCD. The spontaneously breaking of the chiral symmetry, can be achieved by introducing a bifundamental complex scalar field $X(z)$. This field belongs to the adjoint representation of the 5D gauge group $S U\left(N_{f}\right)_{L} \times S U\left(N_{f}\right)_{R}$. To satisfy the linear confinement feature of QCD, one simply has to turn on the holographic coordinate dependent dilaton field $\Phi$ with the limit $\Phi(z \rightarrow \infty) \sim(k z)^{2}$ where the parameter $\mathrm{k}$ sets the meson mass scale. Also the dilaton background assumed to be asymptotically quadratic as, $z \rightarrow \infty$. According to the general rules of gauge/gravity duality, there are two 5D gauge fields $\left(A_{L}\right)$ and $\left(A_{R}\right)$ which are dual to the $4 \mathrm{D}$ chiral currents $J_{L}^{\alpha \mu}=\bar{\psi}_{L}^{\mu} \gamma^{\mu} t^{\alpha} \psi_{L}$ and $J_{R}^{\alpha \mu}=\bar{\psi}_{R}^{\mu} \gamma^{\mu} t^{\alpha} \psi_{R}$. The quark bilinear operator $\bar{\psi}_{L}^{i} \psi_{R}^{j}$ is also an important $4 \mathrm{D}$ operator of chiral symmetry breaking. The holographic coordinate $\mathrm{z}$ which corresponds to the energy scale in the 4D theory, defined within the range $0<z<\infty$. The bulk action which describes mesons sector considering only quadratic parts of the field can be written as:

$$
S_{5}=\int d^{5} x \sqrt{G} e^{-\Phi(z)} \operatorname{Tr}\left[|D X|^{2}-m_{x}^{2}|X|^{2}-\frac{1}{4 g_{5}^{2}}\left(F_{L}^{2}+F_{R}^{2}\right)\right]
$$

where $F_{L}$ and $F_{R}$ are the non-Abelian field strength formed from gauge potentials $\left(A_{L}\right)$ and $\left(A_{R}\right)$ respectively and defined in one form as:

$$
F_{L, R}^{M N}=\partial^{M} A_{L, R}^{N}-\partial^{N} A_{L, R}^{M}-i\left[A_{L, R}^{M}, A_{L, R}^{N}\right]
$$

In the equation (2.1) symbol $D$ is the Yang-Mills covariant derivative containing the gauge fields $\left(A_{L}, A_{R}\right)$ and $m_{x}^{2}=-3$. In the integration measure $G$ denotes the determinant of the metric $\left(D_{M N}\right)$, so $\sqrt{G}=a^{5}$ for the $A d S_{5}$ space. Also the $D_{M} X$ can be written as $D_{M} X=\partial_{M} X-i A_{L M} X+i X A_{R M}$ 
and the gauge coupling $g_{5}$ to be $g_{5}^{2}=\frac{12 \pi^{2}}{N_{c}}=4 \pi^{2}$ and for QCD, $N_{c}=3$. The metric in a 5D AdS geometrical background written as:

$$
d s^{2}=G_{M N} d x^{M} d x^{N}=a^{2}(z)\left(\eta_{\mu v} d x^{\mu} d x^{v}-d z^{2}\right)
$$

where $\eta_{\mu \nu}$ is the 4D Minkowski metric given by: $\eta_{\mu \nu}=\operatorname{diag}(1,-1,-1,-1)$ and $\alpha(z)$ is the conformal factor (or warped factor). The vacuum expectation value (vev), $v(z)$ of the scalar field $\left(\langle X\rangle=\frac{1}{2} v(z)\right)$ assumed to have a z-dependent behavior with limit: $v(z \rightarrow 0) \sim a z+\beta z^{3}$. Using the equation(2.1) the equation of motion (EOM) of the vacuum expectation value in the axial gauge can defined as:

$$
\partial_{z}\left(a^{3} e^{-\Phi} \partial_{z} v\right)+3 a^{5} e^{-\Phi} v=0
$$

The hadrons are defined as a normalizable modes of the $5 \mathrm{D}$ gauge fields. The vector mesons (V) and the axial-vector mesons (A) can be written respectively:

$$
V=\frac{A_{L}+A_{R}}{2}
$$

and

$$
A=\frac{A_{L}-A_{R}}{2}
$$

According to Kaluza-Klein (KK), a dimensional reduction of a 5D vector field, gives rise to a tower of 4D massive vector fields called KK-modes. These KK-modes can be decomposed by breaking the field in an infinite tower of 4D components, satisfying the Proca's equation for massive vector bosons [7] and the extra dimensions dependent parts. The vector meson field (V) can be written then:

$$
V_{\mu}(x, z)=\sum_{n} \rho_{\mu}^{(n)}(x) h_{V}^{n}(z)
$$

where the z-dependent part satisfy the constrain [8]:

$$
-\partial_{5}\left(a e^{-\Phi} \partial_{5} h_{V}^{(n)}\right)=a e^{-\Phi}\left(M_{V}^{(n)}\right)^{2} h_{V}^{(n)}
$$

where $\left(M_{V}^{(n)}\right)^{2}$ are the masses of vector fields $\rho_{\mu}^{(n)}(x)$. The infinite tower of $4 \mathrm{D}$ massive vector fields $\rho_{\mu}^{(n)}(x)$ resulting from KK decomposition of the vector field (V), assumed to be the vector $\rho$-meson of low-energy QCD and the equation (2.7) determines its mass spectrum. Also this equation can be changed by simply setting the $h_{V}^{(n)}=e^{[\Phi(z)-\log a(z) / 2]} \chi_{V}^{(n)}$ to the Shrödinger form:

$$
-\frac{d^{2}}{d z^{2}} \chi_{V}^{(n)}+V_{V} \chi_{V}^{(n)}=\left(M_{V}^{(n)}\right)^{2} \chi_{V}^{(n)}
$$

with potential:

$$
V_{V}(z)=\frac{1}{4}\left[\frac{d}{d z}(\Phi(z)-\log a(z))\right]^{2}-\frac{1}{2}\left[\frac{d^{2}}{d z^{2}}(\Phi(z)-\log a(z))\right]
$$




\section{The dilaton gauge field}

In the following analysis a modified version of soft-wall model according to the dilaton gauge field presented. The model advocated at [9] and modified further by others [10],[11],[12]. About the interesting regions in AdS geometry one can say that a dilaton gauge field can be relay between the IR region with $\Phi \sim z^{2}$ which is just the requirement for the Regge behavior and the UV with $\Phi \sim z$. So the proposed dilaton field actually is a combination between the two aforementioned regions and finally it looks like the sum of their represented fields.

$$
\Phi(z)=(k z)^{2}+k z
$$

This simple modified relation of the dilaton gauge field has the advantage of the positive $\mathrm{z}$-dependent term, which according to [6] is able to avoid massless modes for the vector sector, where the sign of the exponential profile defining the wall should be positive. The dilaton gauge field has one parameter the $k$ and is able to reproduce the correct Regge trajectories behavior. This analysis focus on the $\rho$ vector meson which the most represented meson for that purpose. One to define the meson mass spectra also needs an additional specification of the warped factor. The proposed type of this factor is the following simple:

$$
a(z)=\frac{1}{z}
$$

Using the equation (2.10) one can easy evaluate the Shrödinger-like representation and for the vector potential can have:

$$
V_{V}(z)=k^{4} z^{2}+k^{3} z+\frac{k^{2}}{4}+\frac{1}{2 z}\left[k^{2}+\frac{3}{2 z}\right]
$$

\section{The $\rho$ meson mass spectrum}

To get the $\rho$ meson mass from equation (2.9) one using the potential given at (3.3) make use of boundary conditions $\chi_{n}(z \rightarrow 0)=0$ and $\partial_{z} \chi_{n}(z \rightarrow \infty)=0$ and the value for $\mathrm{k}=402 \mathrm{MeV}$. One doing this calculations can construct the theoretical masses of the $\rho$ meson mass spectra. These masses given in table I, the experimental values for comparison taken from [11].

Table 1: The theoretical and experimental values of the $\rho$ meson. The average error is $5.43 \%$

\begin{tabular}{cccccccc}
\hline \hline$\rho$ & 0 & 1 & 2 & 3 & 4 & 5 & 6 \\
\hline$m_{\text {th }}(\mathrm{MeV})$ & 984 & 1296 & 1543 & 1754 & 1941 & 2139 & 2257 \\
$m_{\text {exp }}(\mathrm{MeV})$ & $775.5 \pm 1$ & $1282 \pm 37$ & $1465 \pm 25$ & $1720 \pm 20$ & $1909 \pm 30$ & $2149 \pm 17$ & $2265 \pm 40$ \\
Error & $27 \%$ & $1.1 \%$ & $5.3 \%$ & $2 \%$ & $1.7 \%$ & $0.5 \%$ & $0.4 \%$ \\
\hline \hline
\end{tabular}

\section{Conclusion}

In this work studied in the frame of the soft-wall AdS/QCD model the behavior of the $\rho$ meson mass spectra using a simple modification of the gauge dilaton background. When this modification 
applied it seems that an improved progress can be achieved. The errors as one can see in Table 1 are small especially for the high masses. Nevertheless this simple modification at the gauge dilaton background field indicates that the model has real behavior and is comparable with the experimental data. Furthermore the positive form of the dilaton gauge field satisfies the constrain for having the correct Regge trajectories. This is an proof that we do not have massless modes in a vector sector.

\section{Acknowledgments}

I am grateful to the organizers of the "6th International Conference in Quark and Nuclear Physics (QNP 2012), 40 years of development of QCD", for the opportunity to present this work.

\section{References}

[1] J. M. Maldacena, "The large N limit of superconformal Field theories and supergravity", Adv. Theor. Math. Phys. 2 (1998) 231-252, [arXiv:hep-th/9711200].

[2] E. Witten, "Anti-de Sitter space and holography", Adv. Theor. Math. Phys. 2 (1998) 253; "Anti-de Sitter space, thermal phase transition, and confinement in gauge theories", ibidem 2 (1998) 505; S. S. Gubser et al., "Gauge theory correlators from non-critical string theory", Phys. Lett. B 428 (1998) 105.

[3] J. Polchinski et al., "Hard scattering and gauge/string duality", Phys. Rev. Lett. 88 (2002) 031601.

[4] S. J. Brodsky and G. F. de Teramond, [arXiv:0802.0514].

[5] O. Andreev, "1/ $q^{2}$ corrections and gauge / string duality", Phys. Rev. D 73 (2006) 107901.

[6] A. Karch et al., Linear confinement and AdS/QCD, Phys. Rev. D 74 (2006) 015005.

[7] Alexander Proca (1897-1955) the great physicist. [arXiv:0508195v1].

[8] Thomas M. Kelley, "The Dynamics and Thermodynamics of Soft-Wall AdS/QCD", [arXiv:hep-ph/1108.0653].

[9] J. Erlich, E. Katz, D. T. Son and M. A. Stephanov, "QCD and a Holographic Model of Hadrons", Phys. Rev. Lett. 95, 261602 (2005) ,[arXiv:hep-ph/0501128].

[10] T. Gherghetta, J. I. Kapusta and T. M. Kelley, "Chiral Symmetry Breaking in Soft-Wall AdS/QCD", Phys. Rev. D79: 076003 (2009), [arXiv:0902.1998].

[11] P. Zhang, "Linear Confinement for Mesons and Nucleons in AdS/QCD", JHEP 05 (2010) 039 [arXiv:1003.0558]; "Mesons and Nucleons in Soft-Wall AdS/QCD", Phys.Rev. D82, 094013 (2010) [arXiv:1007.2163]; "Constraining the Infrared Behavior of the Soft-Wall AdS/QCD Model", [arXiv:1105.6293].

[12] A.Vega and I.Schmidt, "Hadrons in AdS/QCD Correspondence", Phys. Rev. D79,055003 (2009), [arXiv:0811.4638]. 